\title{
Transcriptomic analysis of formation and release of monospores in Pyropia yezoensis (Bangiales, Rhodophyta)
}

\section{Shanshan Song}

Shanghai Ocean University

Xing-Hong Yan ( $D$ xhyan@shou.edu.cn )

Shanghai Ocean University https://orcid.org/0000-0002-0906-1198

\section{Research article}

Keywords: Pyropia yezoensis, Monospores, RNA-Seq, DEGs, Real-time PCR, PyMFG, Chitinase

Posted Date: May 20th, 2019

DOI: https://doi.org/10.21203/rs.2.9612/v1

License: (c) (i) This work is licensed under a Creative Commons Attribution 4.0 International License.

Read Full License 


\section{Abstract}

Background Certain red algae (Pyropia yezoensis) of the genus Pyropia can be asexually reproduced by producing monospores, which provides a large number of secondary seeds for marine production. However, the molecular biological mechanism of how algal regulates its own monospores' formation and release is still unclear, which brings great difficulties and challenges for regulating the yield of seaweed and discovering new strains. Results In this study, we compared and analyzed the data between PY26W' which release a large number of monospores and PY26W which release few monospores by Illumina sequencing platform. The number of DEGs produced by the PY26W' was much higher than that of PY26W, and upregulated genes dominantly in PY26W'. A total of 415 common DEGs were produced between comparison of PY26W and PY26W', which may be involved in formation and release of monospores. All the DEGs were highly enriched in the translation process, ribosome assembly, RNA methylation, assembly of actin and vesicles, intracellular localization of organelles, endocytosis and other biological processes and MAPK signaling pathways. Four DEGs (Contig-21827, Contig-15542, Contig-13390, Trinity-DN39215) were selected for real-time PCR, and the results were highly consistent with the transcriptome sequencing results in both strains, and their expression levels were significant. Further expression analysis indicated that Contig-21827 was abundantly expressed in Pyropia. suborbiculata, Pyropia. chauhanii and PY26W' but not in PY26W and Porphyra haitanensis (WT-8), indicating it plays a key regulatory role in the formation and release of monospores. This gene was further confirmed to be involved in the formation of monospores by analogous conidia formation pathway and was named PyMFG. Conclusion This is the first time to investigate the formation and release of monospores in P. yezoensis from the RNA level. We successfully screened the monospores' formation gene (PyMFG) of P. yezoensis and the chitinase homologous gene that degraded its cell wall of monosporangia. Which lay a theoretical foundation for the subsequent explanation of the molecular biological mechanism of its formation and release, regulation of seaweed yield and discovery of new seaweed strains.

\section{Background}

Pyropia yezoensis [1] is mainly distributed in the Yellow Sea, Bohai Sea and the northern coast of the East China Sea as well as the coast of Japan and the Korean Peninsula, and its production plays an important role in the seaweed industry worldwide. Studies have shown that $P$. yezoensis uses filamentous sporophyte and leafy gametophyte to complete generational alternation. Besides, asexual reproduction in $P$. yezoensis provides a large number of secondary seeds for cultivation in the sea [2]. In the process of conversion into monospores, the vegetative cells of the algae change from red to golden yellow, and then they are released by monosporangia or germinated in situ [3]. With observation on the life history of different seaweeds, individual biomass is increased by producing monospores in Pyropia tenera, Pyropia suborbiculata, Pyropia chauhanii and so on [4-6]. Existing evidence suggests that the formation and release of monospores in Pyropia affect the size and quantity of algae to a certain extent, and determine its yield ultimately [7]. 
Current studies have confirmed that changes in culture conditions, such as light, temperature, seawater density and nutrients, can affect the formation and release of monospores of $P$. yezoensis [8-11].

Furthermore, the addition of exogenous substances, such as allantoin and ammonium sulfate, also has a significant effect on the release of monospores $[10,12,13]$. Early studies have indicated that $P$. yezoensis can produce mutants, which release monospores heavily by induction of methyl nitrosoguanidine [2]. Researchers have used ${ }^{60} \mathrm{Coy}$-rays to induce and separate strain of $P C-Y 1$ that does not release monospores in recent years. Above-mentioned evidence indicates that the biological traits of formation and release of monospores are controlled by genes and can be stably inherited after several generations of cultivation [14].

At present, with the continuous improvement of the individual monospores, it has become the trend of current research to explore the formation and release of monospores at the molecular level. Studies have shown that the amount of released monospores is closely related to the interspecific genetic distance of algae. The closer the genetic distance between the varieties of algae that can release monospores, the smaller the difference in the amount of released monospores [15]. In addition, some studies have pointed out that the molecular weight of cDNA is significantly reduced during the process of vegetative cells specializing in monospores, indicating that the expression of genetic information has been changed [16]. However, the significant expressions of genes involved in the Calvin cycle and the influence of allantoin on the release of monospores also illustrate that the mass formation and release of monospores are closely related to energy and purine metabolic pathways $[13,17]$.

With the maturity and popularity of next-generation sequencing technology, transcriptomics is used to identify key genes controlling specificity in non-reference genomic organisms, to analyze the trend of gene expression during reproductive development, and to explain the complex molecules of specific traits. The mechanism has become a hot topic of current research. Therefore, we, in the present study, aimed to explore the key genes involved in the regulation of sporangium formation and release at the transcriptome level. Our findings provided valuable insights into the molecular mechanism underlying the formation and release of monospores in P. yezoensis.

\section{Methods}

\section{Seaweed materials and monospore release}

PY26W (few monospores) and PY26W' (large-scale monospores) were used in the present study, and both of them were obtained by separating and purifying from the spottedly variegated foliose thalli produced by the hybridization between red mutant (fre-1,囚) which releases monospores heavily and wild type $(U-511, \mathbb{Z})$ which releases few monospores, referred to as $W$ and $W$ 'as follows. Separation and purification were performed as previously reported [18]. The genetic backgrounds of these two strains were similar, while their biological characteristics of monospores were significantly different, which are reliable materials for investigating the formation and release of monospores. In addition, $P$. suborbiculata (PS-WT), P. chauhanii (PC-WT) and P. haitanensis (WT-8) were also used in this experiment. 
Gametophytes of three variety were cultured at $23 \pm 1^{\circ} \mathrm{C}$ under $50 \mu \mathrm{mol}$ photons / $\left(\mathrm{m}^{2} \cdot \mathrm{s}\right)$ with $10 \mathrm{~L}: 14$ $D$ light cycle. All of the above-mentioned algal varieties were saved in the laboratory in the form of freeliving conchocelis filaments, and the preservation was conducted as Liu described [19]. Culture solution used for gametophytes was sterilized seawater, to which MES medium was supplemented [20].

On the 18th day of culture, 500 gametophytes with the same size and growth potential were randomly selected, and then the proportion of gametophytes releasing the monospores within 7 days was counted to confirm the starting age of the transcriptome sequencing. In addition, 30 gametophytes were randomly selected to count the total numbers of monospores per geametophyte during 18-35 days between $W$ and $W$ '. Three biological replicates were set for each group of experiments. First, each group of gametophytes was placed in a white plastic cup for aeration culture. The gametophytes were removed with sterile scorpion on the next day, placed in a new plastic cup containing fresh culture medium and continued to culture. The previously released monospores were brushed down and placed under suitable conditions, and the culture was counted until the gametophyte of each strain was visible to the naked eyes. The culture conditions were as follows: $19 \pm 1{ }^{\circ} \mathrm{C}, 40 \mu \mathrm{mol}$ photons / $\left(\mathrm{m}^{2} \cdot \mathrm{s}\right), 10 \mathrm{~L}$ : $14 \mathrm{D}$. The total amount of monospores released was obtained after 15 days of continuous monitoring, by which the ability of both strains to disperse monospores and the cut-off age of the sequencing samples were confirmed.

Transcriptome sequencing was performed in two comparisons. First, a comparison of samples in each strain was performed to obtain DEGs between day 25 and day 35, and then samples of the same age group were compared to obtain DEGs between $W$ and $W^{\prime}$.

\section{RNA extraction, transcriptome de novo assembly, and annotation}

The gametophytes were washed with distilled water and blotted dry with absorbent paper. Subsequently, the gametophytes with a wet weight of 0.1-0.3 g were placed in liquid nitrogen and rapidly ground, and their total RNA was extracted with Trizol reagent. The absorbance at wavelengths of $260 \mathrm{~nm}$ and $280 \mathrm{~nm}$ (A260/A280) was determined by NanoDrop 2000, respectively, and the integrity was examined by $1 \%$ agarose gel electrophoresis and Agilent 2200. After the RIN was confirmed to be greater than 6.0 in all sequencing samples, $2 \mu \mathrm{g}$ or more RNA was used to construct a cDNA library.

RNA sequencing was performed by the Illumina HiSeqXTen sequencing platform (NovelBio Bio-Pharm Technology Co. Ltd, Shanghai). Sequencing data were analyzed using the following processes. Firstly, the Fast-QC (http://www.bioinformatics.babraham.ac.uk/projects/fastqc/) was used to perform an overall quality assessment of the raw sequencing data, and then the Trinity software was employed to perform de novo clean reads on a single sample. Furthermore, Cap3 was used to merge the assembly results to eliminate redundancy. The spliced sequences were then separately aligned to Arabidopsis thaliana, Aspergillus nidulans, Saccharomyces cerevisiae, Bacillus subtilis, Chlamydomonas and Porphyra purpurea to identify the homologous sequence between $P$. yezoensis and model species in order to 
determine the genetic annotation and functional prediction. Finally, mapping was performed using BWA software and BWA-MEM strategy, FPKM values were calculated, and the gene expression was reflected based on the FPKM values.

\section{Differential expression analysis}

In order to obtain the expression pattern of a certain differential gene, DEGs were screened by comparison between $W$ or $W^{\prime}$ using the EBSeq algorithm by setting the criteria for differential screening at $P$ -

value $<0.05, F D R \leq 0.05$ and $\log _{2}$ |fold change $\geq 1$. FDR and P-value were used to control the accuracy of comparison among multiple samples.

To further verify the gene expression at the mRNA level, four DEGs were selected for qRT-PCR verification according to the FPKM value $\geq 1$ in $W^{\prime} 35 d$, the FPKM values of the other three samples were $<1$, and the primers were designed in the open reading frame region using NCBI Primer Blast (Table 1). Firstly, RNA of each sample was reversely transcribed into CDNA using Takara reverse transcription kit (Takara, Japan). Secondly, qRT-PCR was performed with Takara's SYBR Green fluorescent dye and Bio-Rad CFX-96 realtime PCR system using cDNA as a template and $\mathrm{PH} 18 \mathrm{~S}$ as a reference gene. Three biological replicates were set for each gene, and three technical replicates were set for each biological replicate. The relative expression level of each gene was calculated according to the $2^{-\Delta \Delta C t}$ method [13]. The program setting of qRT-PCR system was the same as previously described [21]. The experimental data were statistically analyzed using Origin 8.5 software, and the differences between the different data groups were compared by one-way ANOVA and LSD test. $P<0.05$ indicates significant difference.

\section{Gene expression pattern analysis and functional enrichment of DEGs}

The consensus DEGs obtained by comparing the strains ( $W$ vs. $W$ ) were GO-annotated based on the gene function annotation database to obtain all GO terms in which the genes participated. Then the significant GO terms were screened by Fisher's exact test. In addition, pathway annotations were performed based on the KEGG database to obtain all pathways. Then the Fisher's exact test of hypergeometric distribution was used to screen the significantly enriched pathways. The criterion for significant screening was Pvalue $<0.05$.

\section{Statistical analysis}

Relevant statistical analysis was carried out using $\mathrm{R}$ language-related packages.

\section{Results}




\section{Release of monospores}

We found that the proportion of the gametophytes releasing monospores was continuously increased after the $W^{\prime}$ strain was cultured for 18 days, while such proportion at the 25 th day did not exceed $30 \%$, and the overall proportion was relatively small (Fig. 1a). Within 18-35 days, the total amount of monospores in each gametophyte of the $W^{\prime}$ strain could be as high as 1,100, and the amount of scatter was relatively large (Fig. 1b). However, only two monospores were found in the $W$, which released less monospores (Fig. 1a, Fig. 1b). Therefore, the time points of the two strains for sequencing were determined to be 25 day and 35 day, respectively.

\section{Data quality control and de novo assembly}

Library sequencing was performed on $W^{\prime} 25 d, W^{\prime} 35 d, W 25 d$ and $W 35 d$ samples by the lllumina HiSeqXTen sequencing platform. The number of clean reads produced by each sample was $46,327,654$, $33,036,768,39,605,176$ and $34,710,324$, respectively, and the GC content was above $60 \%$. A total of 53,689 contigs were obtained from de novo assembly and long sequence clustering. The ratio of contigs mapped to Arabidopsis thaliana, Aspergillus nidulans, Saccharomyces cerevisiae, Bacillus subtilis, Chlamydomonas and Porphyra purpurea was higher than $99 \%$. The number of mapped unigenes was $25,623,907,20,102,090,21,395,041$ and $18,685,953$, respectively, and the ratio of mapped unigenes was higher than $50 \%$ (Table 2).

\section{Statistical analysis of DEGs}

Based on the FPKM values, four groups of DEGs were analyzed. The number of DEGs was 4,705 ( $W^{\prime} 35 \mathrm{~d}$ vs. $W^{\prime} 25 d$ ), 2,365 (W35d vs. $W 25 d$ ), 3,031 ( $W^{\prime} 25 d$ vs. $W 25 d$ ) and 635 ( $W^{\prime} 35 d$ vs. $W 35 d$ ). The results showed that in the comparison of sampling time, the number of DEGs produced in $W^{\prime}$ was the highest, and the DEGs were mainly up-regulated. However, the number of DEGs in $W$ was approximately the same. In the comparison between $W$ and $W$, the number of DEGs at 25 days was higher than that at 35 days, but the proportion of up-regulated DEGs at 35 days was larger (Fig. 2). Moreover, the number of DGEs in $W$ during the 25-35 days of culture was significantly less than that in $W$ '. All FPKM values in different samples were shown in Additional file 1: Table S1.

In addition, the consensus DEG analysis showed that there were 415 shared DEGs in the two comparison groups between $W$ and $W^{\prime}$, while only 64 DEGs were found in the comparison group in the same strain (Fig. 3). Original specific statistics was shown in Additional file 2: Table S2.

\section{Analysis of GO and pathway enrichment of DEGs}


There were 415 DEGs between the $W$ and $W^{\prime}$ comparison groups at the same age, and these genes might be involved in the formation and release of monospores. $\mathrm{GO}$ enrichment analysis showed that its function was highly enriched in biological processes, such as translation, RNA activity, actin and vesicle assembly, intracellular localization of Golgi and mitochondria, and peroxisome activity. Among them, the number of DEGs involved in translation, RNA methylation and ribosome assembly was high (Fig. 4). Moreover, KEGG analysis revealed that the above-mentioned DEGs were mainly enriched in ribosome assembly, endocytosis, amino acids (Gly/Ser/Arg/Val), carbon and nitrogen metabolic pathways, photooxidation, as well as AGE-RAGE and MAPK signaling pathways (Fig. 5). Raw data of GO and Pathway analysis can be seen in Additional file 3: Table S3 and Additional file 4: Table S4.

\section{Analysis of expression patterns of candidate DEGs}

Comprehensive genome annotation, GO enrichment (Fig. 4) and pathway signaling pathway analysis (Fig. 5) were further employed to screen some DGEs for expression pattern analysis. Hierarchical cluster analysis showed that the gene types mainly included acyl-CoA dehydrogenase gene, Rab protein family gene, myocardium, myosin gene, ubiquitin ligase gene, chitinase gene and $a b a A$ and $f l b D$ in the genome of $A$. nidulans. Except for the higher expression level of the ubiquitin ligase gene in $W 25 \mathrm{~d}$, the expression levels of all above-mentioned DEGs in $W^{\prime}$ were significantly higher than those in $W$ (Fig. 6).

\section{Verification of DEGs by qPCR and identification of the monospores formation gene}

According to the hierarchical clustering results and the conidia formation pathway in A. nidulans, four major DEGs, including Contig-21827, Contig-15542, Contig-13390 and Trinity-DN39215, were verified by qRT-PCR, which were homologous to $a b a A, f l b D, A N 1339.2$ and AN1966.2, respectively. The results were generally consistent with RNA-Seq data. Besides, the relative expressions of these four genes in $W^{\prime}$ were significantly different from those of the $W$ (Fig. 7). The expression of Contig-21827 in different speciesof Pyropia and strains was further analyzed. The results showed that it was abundantly expressed in $P$. yezoensis $(W)$, $P$. suborbiculata and $P$. chauhanii, and the difference was significant among species. However, it was not expressed in the $P$. haitanensis (WT-8) and $P$. yezoensis (W) (Fig. 8). These results suggested that the function of this gene was related to the formation and release of monospores.

\section{Discussion}

The monospores of Pyropia are developed by mitochondrial specialization of vegetative cells on the gametophyte. The new individuals germinated from monospores carry the same genetic information as the vegetative cells. Besides, individual monospores will independently develop into full leafy gametophytes. This trait is very similar to the formation of conidia. The conidia are Ascomycota fungi which are developed by mitotic development of specialized conidiophores. The newly developed haploid 
individuals have the same genotype as the conidiophore, and they can be released into the proper environment and develop into two separate fungal individuals. We surprisingly found that the Contig21827 sequence was homologous to the key gene $a b a A$ of the conidia formation pathway in $A$. nidulans, and the qRT-PCR results confirmed that the expression of Contig-21827 was highly consistent with the macroscopic statistical results (Fig. 1, Fig. 7, Fig. 8). Therefore, the genes involved in the traits of monospore could be found in the pathway of maturity and release of fungal conidia. Furthermore, the molecular mechanism of the interaction could be elucidated.

\section{Formation of spores}

After de novo reassembly and genome annotation of $A$. nidulans, the homologous sequences of fluG, $f l b A, f l b C, f l b D$ and $a b a A$ existed during the formation and release of monospores in $P$. yezoensis. FluG is the key upstream activator of the central regulatory pathway of $A$. nidulans, which regulates the formation of conidia by sequentially activating SfgA and Flbs (A/B/C/D/E), ultimately acting on BrlA, AbaA and WetA [22-24]. Previous studies have confirmed that the specific expression of $a b a A$ promotes the production of conidial head and maintains conidial production [25-27]. BrlA is located upstream of $a b a A$, which can induce the specific expressions of $a b a A$ and wet $A$ without the involvement of upstream activators to produce fluffy conidial heads. WetA is located downstream of $a b a A$, which regulates the conidium maturation and synthesizes trehalose to maintain normal conidial walls [27-29]. According to the conidial formation pathway and the expression of Contig-21827 in different species of Pyropia, it was preliminarily believed that the gene was involved in the formation of monospores, and it was named PyMFG. However, based on the current annotation information, the homologous sequences of the brlA and wet $A$ genes were not found in $P$. yezoensis, indicating that the formation process of monospores in $P$. yezoensis might not be exactly the same as the regulation of the formation of $A$. nidulans.

\section{Vesicle transport}

Recent studies have found that GTPase-binding proteins involved in $A$. thaliana secreted protein trafficking are highly homologous to the Ypt protein family in S. cerevisiae [30]. Among them, the Rab, Arf and Ras GTP protein kinase subfamilies mediate intracellular vesicle trafficking through interaction with GEFs [31]. However, the intracellular vesicle trafficking process is mainly mediated by Sec and Ypt proteins in yeast. Ypt1p regulates the secretion and fusion of secreted proteins of $S$. cerevisiae, Ypt31p and Ypt32p are responsible for the transport of secretory vesicles inside the Golgi. Sec4p mediates Golgi vesicle transport to the cytoplasmic membrane. However, Ypt7p mediates vacuolar fusion during the in vitro ligation of homozygous yeasts [32-37]. In the present study, the Rab/Ypt homologous protein family genes were found in comparison with the genomes of $A$. nidulans and $S$. cerevisiae. These genes were abundantly expressed in $W^{\prime}$ (Fig. 6). Otherwise, the expressions of DEGs generated by the comparison

between $W$ and $W^{\prime}$ were significantly enhanced in translation, RNA activity, assembly of actin and vesicles, and biological processes, such as Golgi and mitochondria, peroxisome activity, and endocytosis 
(Fig. 4, Fig. 5), which was consistent with the study at the cellular level [16]. Therefore, we speculated that $P$. yezoensis transported functional proteins required for various life activities in the form of vesicles, thereby achieving the effect by rapidly coping with its own stress about the formation and release of monospores.

\section{System of signaling pathway}

The $\mathrm{Ca}^{2+}$ signaling pathway mainly relies on $\mathrm{Ca}^{2+} / \mathrm{CaM}$ and calcineurin to initiate intracellular responses and participate in a variety of biological processes in eukaryotes [38, 39]. Collectotrichum. gloeosprioides and $C$. trifolii begin to express the cam early in sporulation [40]. In addition, the endogenous $\mathrm{Ca}^{2+}$ signaling pathway regulates the germination and fusion of Neurospora crassa germ tube, which is closely associated with its intact colony morphology [41]. We found that there were a large number of upregulated calmodulin homologous genes in $W^{\prime}$ (Fig. 6), which fully demonstrated that the formation and release of monospores activated $\mathrm{Ca}^{2+}$ signaling pathways in cells, thereby stimulating the transmission of CaM to extracellular signals. Previous study has indicated that under normal irradiance, the monosporangia of $P$. yezoensis can influx low-level $\mathrm{Ca}^{2+}$ and induce intracellular signaling pathway to produce a large amount of $\mathrm{IP}_{3}$, which promotes the formation and release of a large amount of monospores, resulting in serious disintegration in algae [42]. However, the absence of midA leads to the imbalance of mannan and chitin components in the cell wall according to the study of midA mutant of $A$. nidulans, by which conidia are forced to flow out [43]. We suspected that the $\mathrm{Ca}^{2+}$ influx involved in the initial germination of monospores activated the $\mathrm{Ca}^{2+}$ signaling pathway, which eventually caused changes in cell wall components and cytoskeleton, thereby achieving monospores.

As a Ser/Thr protein kinase, MAPK plays a key role in gene expression and cytoplasmic activity. Sequencing data showed that the MAPKK kinase gene was highly enriched in the sample $W^{\prime} 35 \mathrm{~d}$ (Fig. 6), and the MAPK signaling pathway was significantly enriched in the top 20 significant signaling pathways (Fig. 5). At present, the amplification reaction of MAPK cascade in animal and yeast systems can produce a variety of stress responses by regulating the expressions of related genes [44]. In addition, many biological processes, such as spore formation pathway, cell wall integration pathway (MAPK CWI), Rho/Ras signaling pathway, cytoskeletal movement, vesicle trafficking, endocytosis, transcription and translation of $N$. crassa, are inseparable from MAPK [41, 45]. Therefore, we speculated that the MAPK signaling pathway might have a certain regulatory effect on the formation and release of monospores in Pyropia.

\section{Cytoskeletal movement}

The narrow cytoskeleton mainly refers to microfilaments, microtubules and intermediate fibers, and the combination of actin and myosin can achieve cell movement in animals or cytoplasmic flow in plants. During the transformation of the vegetative cells of $P$. yezoensis into monospores, the morphology of cell 
gradually becomes rounded by regular polygons, and then the plasmolysis occurs in cell, leading to the separation of protoplasts from the cell wall in the form of "water drop", and leaving only a transparent gelatinous layer. The polar growth of blade is rapidly established after encountering suitable environment $[46,47]$. Studies have shown that certain red algae (Porphra umbilicalis et al.) of Porphyra have fewer cytoskeletal components and lower complexity, which limits the development of vegetative cells and prevents them from growing into large plants with multicellular structures. In addition, the cytoplasmic flow of cells is also severely blocked due to the lack of dynein and multiple kinesin family genes [48]. However, algae in the Pyropia genus capable of dispersing monospores can achieve cytoplasmic flow and cell morphological changes in the formation of monospores. We also found that some actin and myosin homologous genes were highly expressed in $W$ ', suggesting that the intracellular components and assembly complexity of actin and myosin in this type of algae were higher than those of Porphyra genus. Therefore, it could regulate the morphological changes of vegetative cells and cause the separation of protoplasts.

\section{Hydrolase of cell wall}

The current research has confirmed that the conchase and agarase isolated from marine animals and microorganisms, such as conch, sarcophagus and Pseudomonas, can effectively decompose the cell wall of vegetative cells of algae $[49,50]$. However, it remains unclear whether the algae itself can produce enzymes to degrade cell wall. We successfully screened the chitinase homologous gene based on the genome of $A$. nidulans in $P$. yezoensis. Besides, the expression of this gene was significantly increased in $W^{\prime} 35 \mathrm{~d}$ samples with a large number of monospores (Fig. 6). Chitinase belongs to the family of glycosyl hydrolases, which can effectively decompose the conidial wall of fungi with chitin and polysaccharide as main components to achieve spore development and cell autolysis [51-56]. In addition, the FluG-BrlA feedback pathway of $A$. nidulans auxotrophic strains can also produce chitinase to decompose cell walls and organelles to autolyze cells, leading to further release of conidia $[28,56]$. Rac-1 gene in N. crassa breaks down plant cell walls by synthesizing polysaccharide hydrolases, such as lignocellulase [57-61]. All of above-mentioned evidence indicated that the chitinase homologous gene in $P$. yezoensis was very likely to be involved in the degradation of the cell wall of monospoorangia, thus accelerating the release of monospores.

\section{Conclusions}

The transcriptome data suggested that more DEGs were obtained in $W^{\prime}$ than $W$ in comparison group of different age, and these DEGs were mainly up-regulated. A comparison between $W^{\prime}$ and $W$ strains revealed that more DEGs were identified in 25-day samples, the proportion of up-regulated DEGs in 35-day samples was higher, exhibiting obvious trait of monospore (Fig. 2). Besides, the consensus DEGs in the comparison group between $W$ and $W^{\prime}$ (Fig. 3) were involved in biological processes, such as translation, actin and vesicle assembly, ribosome activity, endocytosis, phagosomes and peroxisomes, and these 
DEGs were significantly enriched in pathways, such as MAPK (Fig. 4, Fig. 5), indicating that multiple physiological processes and regulation of the different pathways might play an important role in the formation of monospores. Furthermore, we successfully obtained the PyMFG gene and the chitinase homologous gene that might be involved in monosporangia degradation of the cell wall based on genome annotation and qRT-PCR. These results further widened our understanding of specific molecular biological mechanisms underlying the formation and release of monospores, and provided valuable insights into the future screening and breeding of Pyropia cultivars.

\section{Abbreviations}

PY26W. W; PY26W: W; RIN: RNA integrity number; FPKM: Fragments per kilobase million; FDR: False discovery rate; ANOVA: Analysis of variance; LSD: Least significant difference; DEGs: Differentially expressed genes; GEFs: GMP exchange factor; CaM: Calmodulin; MAPK: Mitogen-activated protein kinase.

\section{Declarations}

\section{Ethics approval and consent to participate}

Not applicable

\section{Consent for publication}

\section{Not applicable}

\section{Availability of data and materials}

All RNA sequencing data were submitted to NCBI SRA database (https://www.ncbi.nlm.nih.gov/sra/) under study number SRP191712. Accession number of each data is as follows: SRR8873477; SRR8873478; SRR8873475; SRR8873476.

\section{Competing interests}

The authors declare that they have no competing interests.

\section{Funding}


We would like to thank following agency's for funding reagents, algal facility costs and personnel expenses: National Key Research and Development Program of China (2018YFD0900606), National Natural Science Foundation of China (31072208), Major Science and Technology Specific Program of Zhejiang Province (2016C02055-6), Science and Technology Planning Project of Jiangsu Province, China (BE2018335), and Open Program of Key Laboratory of Cultivation and High-value Utilization of Marine Organisms in Fujian Province (2017fjscq02). All above funding bodies had no role in the design of the study, the collection, analysis, and interpretation of the data or in writing the manuscript.

\section{Authors' contributions}

Conceived and designed the experiments: Shanshan Song, Xinghong Yan.

Performed experiments: Shanshan Song.

Analyzed the data: Shanshan Song.

All authors have read, edited and approved the final version of the manuscript.

\section{Acknowledgements}

We thank Qingjie Zhang for technical assistance with experiments; Longchen Tang, Linbin Huang for suggestions about writing this article; and Dahai Gao for modifying this article. We thank Hongchang Ding and Xiuwen Yang for helpful guidline during submission.

\section{Reference}

1囚Sutherland JE, Lindstrom SC, Nelson WA, Brodie J, Lynch M D.J, Hwang NS, Choi HG, Miyata M, Kikuchi N, Oliveira MC, Farr T, Neefus C, Mols-Mortensen A, Milstein D, M. Miiler K. A new look at an ancient order: Generic revision of Bangiales (Rhodophyta). J Phycol. 2011; 47(5): 1131-1151.

$2 \varangle$ Yan XH, Fujita Y and Aruga Y. High monospore producing mutants obtained by treatment with MNNG in Porphyra yezoensis Ueda (Bangiales, Rhodophyta). Hydrobiologia. 2004; 512(1-3): 133-140.

3区Ma JH. Studies on monospores and gametophytes of shell seaweed. J Fish Sci Chin. 1996; 20(2): 132138.

4『Zeng CK, Zhang DR. Studies on Porphyra I. Life History of Pyropia tenera. Botanical Sinica. 1954; 3(3): 287-302.

5囚Yang XW, Ding HC, Yan XH Liu CJ. Genetic analysis of the meiosis and morphogenesis of Pyropia suborbiculata. Oceanol Limnol Sinica. 2019; 50(1): 173-180. 
6खSong JT, Yan XH. Cytological observation of Pyropia chauhanii. J Fish Chin. 2015; 39(10): 1479-1486.

$7 \llbracket$ Ying LS. The ecological characteristics of monospores of Porphyra yezoensis Ueda and their use in cultivation. Eleventh International Seaweed Symposium. 1984. p.255-258.

8هLi SY, Wang JC. Effects of irradiation intensity on the formation, dispersion and attachment of monopores in Porphyra yezoensis. Mar Sci. 1984; 8(2): 41-43.

9『Li SY, Xu W, Wang M. Effects of seawater density on the dispersion and attachment of spores of Porphyra yezoensis. Mar Sci. 1986; 10(6): 38-40.

10खLi SY. Effects of ammonium sulfate on the growth and the formation, dispersion and attachment of monospores in Porphyra yezoensis. Mar Sci. 1987; 11(6): 35-39.

11هLi SY, Xu W, Wang M. Effects of irradiation time on the growth of gametophytes and the formation, release and attachment of monospores in Porphyra yezoensis. Mar Sci. 1988; 12(4): 58-61.

$12 \varangle$ Mizuta H, Yasui H, Saga N. A simple method to mass produce monospores in the thallus of Porphyra yezoensis Ueda. J Appl Phycol. 2003; 15(4): 351-353.

$13 \llbracket$ Sun D, Ding HC Yan XH. Effects of allantoin on the release of monospores and differentiation of somatic in Pyropia chauhanii. J Fish Chin. 2018; 42(4): 534-543.

$14 \llbracket$ Zang C, Yan XH. Isolation and characterization of new strains of Pyropia chauhanii (Bangiales, Rhodophyta). J Fish Chin. 2014; 38(9): 1457-1465.

15区Mei JX, Fei XG, Wang B. Study on monospore production of Porphyra yezoensis. Oceanol Limnol Sinica. 2001; 32(4): 402-407.

16खXu L. Ultrastructural and Molecular Biology of monospore formation in Porphyra yezoensis. J Fish Chin. 2003; 27(6): 519-527.

17『Kitade Y, Asamizu E, Fukuda S, Nakajima M, Ootsuka S, Endo H, Tabata S, Saga N. Identification of genes preferentially expressed during asexual sporulation in Porphyra yezoensis gametophytes (Bangiales, Rhodophyta). J Phycol. 2008; 44(1): 113-123.

18囚Liu MJ, Huang LB, Yan XH. Screening and characterization of an excellent species of intraspecific hybrids of Porphyra yezoensis. J Fish Sci Chin. 2015; 22(1): 33-43.

19『Kato M. Comparative studies on the growth and photosynthesis of the pigmentation mutants of Porphyra yezoensis in laboratory culture. Jpn J Phycol. 1984; 32: 334-347.

20『Wang SJ, Zhang XP, Xu ZD Zhang YL. Studies on vegetative cells and protoplast culture of Porphyra haitanensis I. Oceanol Limnol Sinica. 1986; 17(3): 217-221. 
21区Koramutla MK, Aminedi R, Bhattacharya R. Comprehensive evaluation of candidate reference genes for qRT-PCR studies of gene expression in mustard aphid, Lipaphis erysimi (Kalt). Sci Rep. 2016; 6: 25883.

22खEtxebeste O, Garzia A, Espeso EA Ugade U. Aspergillus nidulans asexual development: making the most of cellular modules. Trends Microbiol. 2010; 18(12): 569-576.

23凶Mirabito PM, Adams TH, Timberlake WE. Interactions of three sequentially expressed genes control temporal andspatial specificity in Aspergillus development. Cell. 1989; 57(5): 859-868.

24囚Park HS, Yu JH. Genetic control of asexual sporulation in filamentous fungi. Curr Opin Microbiol. 2012; 15(6): 669-677.

25खClutterbuck A. A mutational analysis of conidial development in Aspergillus nidulans. Genetics. 1969; 63(2): 317.

26『Sewall TC, Mims CW, Timberlake WE. abaA controls phialide differentiation in Aspergillus nidulans. Plant Cell. 1990; 2(8): 731-739.

27凶Tao L, Yu JH. AbaA and WetA govern distinct stages of Aspergillus fumigatus development. Microbiol. 2011; 157(Pt2): 313-326.

28هBao LF, Qin YQ, Qu YB. Progress on the mechanism of FluG-BrlA pathway participating in asexual development of Aspergillus nidulans. Bulletin of Microbiol. 2014; 41(1): 104-110.

29凶Marshall M, Timberlake WE. Aspergillus nidulans wetA activates spore-specific gene expression. Mol Cell. 1991; 11(1): 55-62.

30凶Andersson MX, Sandelius AS. A chloroplast-localized vesicular transport system: a bio-informatics approach. BMC Genomics. 2004; 5(1): 40.

31区ltzen A, Goody RS. GTPases involved in vesicular trafficking: structures and mechanisms. Semin Cell Dev Biol. 2011; 22(1): 48-56.

32खJedd G, Richardson C, Litt R, Segev N. The Yptl GTPase is essential for the first two steps of the yeast secretory pathway. J Cell Biol. 1995; 131(3): 583-590.

33囚Sacher M, Barrowman J, Wang W, Horecka J, Zhang Y, Pypaert M. Ferro-Novick S. TRAPP I implicated in the specificity of tethering in ER-to-golgi transport. Mol Cell. 2001; 7(2): 433-442.

34『Benli M, Döring F, Robinson DG, Yang X, Gallwitz D. Two GTPase isoforms, Ypt31p and Ypt32p, are essential for Golgi function in yeast. EMBO J. 1996; 15(23): 6460-6475.

35区Suvorova ES, Duden R, Lupashin VV. The Sec34/Sec35p complex, a Ypt1p effector required for retrograde intra-Golgi trafficking, interacts with Golgi SNAREs and COPI vesicle coat proteins. J Cell Biol. 
2002; 157(4): 631-643.

36囚Salminen A, Novick PJ. A ras-like protein is required for a post-Golgi event in yeast secretion. Cell. 1987; 49(4): 527-538.

37凶Mayer A, Wickner W. Docking of yeast vacuoles is catalped by the Ras-like GTPase Ypt7p after symmetric priming by Secl8p (NSF). J Cell Biol. 1997; 136(2): 307-317.

38 $₫$ Klee $\mathrm{CB}$, Crouch TH, Krinks MH. Calcineurin: a calcium- and calmodulin-binding protein of the nervous system. Prol Natl Acad Sci U S A. 1979; 76(12): 6270-6273.

39凶Klee CB, Ren H, Wang X. Regulation of the Calmodulin-stimulated Protein Phosphatase, Calcineurin. J Biol Chem. 1998; 273(22): 13367-13370.

40『Kim YK, Li DX, Kolattukudy PE. Induction of $\mathrm{Ca}^{2+}$-calmodulin signaling by hard-surface contact primes Colletotrichum gloeosporioides conidia to germinate and form appressoria. J Bacteriol. 1998; 180(19): 5144-5150.

$41 \llbracket$ Chang CC. $\mathrm{Ca}^{2+} /$ Calmodulin signalling during colony initiation in Neurospora crassa. University of Edinburgh. 2015.

42खMikami K, Li L, Takahashi M, Saga N. Photosynthesis-dependent $\mathrm{Ca}^{2+}$ influx and functional diversity between phospholipases in the formation of cell polarity in migrating cells of red algae. Plant Signal Behav. 2009; 4(9): 911-913.

$43 \llbracket$ Cao JL. Functional characteristics of calcium channel protein MidA in Aspergillus nidulans. Nanjing Normal University. 2011.

44凶Widmann C, Gibson S, Jarpe MB, Johnson GL. Mitogen-activated protein kinase: conservation of a three-kinase module from yeast to human. Physiol Rev. 1999; 79(1): 143-180.

45凶Rodríguez-Peña JM, García R, Nombela C, Arroyo J. The high-osmolarity glycerol ( $\mathrm{HOG}$ ) and cell wall integrity (CWI) signalling pathways interplay: a yeast dialogue between MAPK routes. Yeast. 2010; 27(8): 495-502.

46هLi L, Saga N, Mikami K. Phosphatidylinositol 3-kinase activity and asymmetrical accumulation of Factin are necessary for establishment of cell polarity in the early development of monospores from the marine red alga Porphyra yezoensis. J Exp Bot. 2008; 59(13): 3575-3586.

47هLi L, Saga N, Mikami K. Ca ${ }^{2+}$ influx and phosphoinositide signalling are essential for the establishment and maintenance of cell polarity in monospores from the red alga Porphyra yezoensis. $\mathrm{J}$ Exp Bot. 2009; 60(12): 3477-3489. 
48囚Brawley SH, Blouin NA, Ficko-Blean E, Wheeler GL, Lohr M, Goodson HV, Jenkins JW, Blaby-Haas CE, Helliwell KE, Chan CX, et al. Insights into the red algae and eukaryotic evolution from the genome of Porphyra umbilicalis (Bangiophyceae, Rhodophyta). Prol Natl Acad Sci U S A. 2017; 114(31): E6361E6370.

49هZhao Y, Zhang N, Li BW, Huang Q, Chen YX. Comparison of enzyme activity of algae from three kinds of snail sources. J Xiamen Univ (Natural Science). 2005; 44(2): 276-278.

50खHan BQ, Liu WS, Wang H, Dai JX. Studies on the enzymes of seaweed cleavage. Mri Sci. 1997; 21(3): 47-49.

51区Adams DJ. Fungal cell wall chitinases and glucanases. Microbiol. 2004; 150(7): 2029-2035.

52₫Gooday GW, Zhu WY, O'Donnell RW. What are the roles of chitinases in the growing fungus? FEMS Microbiol Lett. 1992; 100(1-3): 387-391.

53खPocsi I, Leiter E, Kwon NJ, Shin KS, Kwon GS, Pusztahelyi T, Emri T, Abuknesha RA, Price RG, Yu JH. Asexual sporulation signalling regulates autolysis of Aspergillus nidulans via modulating the chitinase ChiB production. J Appl Microbiol. 2009; 107(2): 514-523.

54『Reyes F, Calatayud J, Martinez MJ. Chitinolytic activity in the autolysis of Aspergillus nidulans. FEMS microbiol lett. 1988; 49(2): 239-243.

55囚Reyes F, Calatayud J, Martinez MJ. Endochitinase from Aspergillus nidulans implicated in the autolysis of its cell wall. FEMS microbiol lett. 1989; 60(1): 119-124.

56هYamazaki H, Yamazaki D, Takaya N, Takagi M, Ohta A, Horiychi H. A chitinase gene, chiB, involved in the autolytic process of Aspergillus nidulans. Curr Genet. 2007; 51(2): 89-98.

57凶Coradetti ST, Craig JP, Xiong Y, Shock T, Tian C, Glass NL. Conserved and essential transcription factors for cellulase gene expression in ascomycete fungi. Prol Natl Acad Sci U S A. 2012; 109(19): 7397-7402.

$58 \llbracket$ Sun J, Tian C, Diamond S, Glass NL. Deciphering transcriptional regulatory mechanisms associated with hemicellulose degradation in Neurospora crassa. Eukaryot Cell. 2012; 11(4): 482-493.

59هBenz JP, Chau BH, Zheng D, Bauer S, Glass NL, Somerville CR. A comparative systems analysis of polysaccharide-elicited responses in Neurospora crassa reveals carbon source-specific cellular adaptations. Mol. Microbiol. 2014; 91(2): 275-299.

60खShen WC, Wieser J, Adams TH, Ebbole DJ. The Neurospora rca-1 gene complements an aspergillus flbD sporulation mutant but has no identifiable role in Neurospora sporulation. Genetics. 1998; 148(3): 1031-1041. 
61هWieser J, Adams TH. flbD encodes a Myb-like DNA-binding protein that coordinates initiation of Aspergillus nidulans conidiophore development. Gene Dev. 1995; 9(4): 491-502.

\section{Tables}

Table 1 Primers of qRT-PCR about DEGs during monospores formation and release in P. yezoensis.

\begin{tabular}{cccc}
\hline Gene ID & Primers & Primer sequences (5'-3') & Annealing $/{ }^{\circ} \mathrm{C}$ \\
& & & \\
\hline Contig-21827 & $\mathrm{F}$ & CAGAAGAACACATTGCAGAACGA & 55.3 \\
& $\mathrm{R}$ & GTGATGGTAAACCTGCAACGG & 56.8 \\
Contig-15542 & $\mathrm{F}$ & CAACCACTGGTCGTTGGTCT & 57.8 \\
& $\mathrm{R}$ & CGACCTTGCATTTTTCAGC & 55.8 \\
\hline Trinity-DN39215 & $\mathrm{F}$ & TACGCAATCTTCCTCGCGTT & 56.8 \\
& $\mathrm{R}$ & CACGTCGTAGAATACGCATGG & 56.0 \\
\hline Contig-13390 & $\mathrm{F}$ & ACTCCTGGGGAGATGGTGATT & 58.0 \\
& $\mathrm{R}$ & ATGCATTGGCCCTGTATGGT & 57.1 \\
\hline PH18S & $\mathrm{F}$ & GTCCAGAGCGCTTTGAGATG & 59.8 \\
& $\mathrm{R}$ & AACCCTAATTCCCGTCACC & 59.8 \\
\hline
\end{tabular}

Table 2 Assessment of transcriptome sequencing data quality and gene alignment rate in $P$. yezoensis. 


\begin{tabular}{|c|c|c|c|c|}
\hline Sample name & $W^{\prime} 25 d$ & $W^{\prime} 35 \mathrm{~d}$ & $W 25 \mathrm{~d}$ & $W 35 \mathrm{~d}$ \\
\hline TotalReads_Before & 55275334 & 39534000 & 48001920 & 41619454 \\
\hline TotalReads_After & 46327654 & 33036768 & 39605176 & 34710324 \\
\hline GC\%_Before & 65.5 & 61 & 65 & 65 \\
\hline GC\%_After & 65 & 60 & 64.5 & 64.5 \\
\hline Total Contig & \multicolumn{4}{|c|}{53689} \\
\hline Mapped & 46168793 & 32836296 & 39443646 & 34629042 \\
\hline Mapped Rate/\% & 0.997 & 0.994 & 0.996 & 0.998 \\
\hline Unigene Mapped & 25623907 & 20102090 & 21395041 & 18685953 \\
\hline Unigene Mapped Rate/\% & 0.553 & 0.608 & 0.54 & 0.538 \\
\hline
\end{tabular}

\section{Additional File Legend}

Additional file 1: Table S1. All fpkm values generated in our four sequencing samples. (xlsx 8,623kb)

W-25D-2 represents $W^{\prime} 25 \mathrm{~d}$; W-35D-3 represents $W^{\prime} 35 \mathrm{~d}$; W-25D-4 represents $W 25 \mathrm{~d}$; W-35D-5 represents $W$ $35 \mathrm{~d}$, which consistent with the following additional files. Additional file 2: Table S2. DEGs generated by comparisons between $W$ and $W^{\prime}$. (xlsx 117kb)

Additional file 2: Table S2. DEGs generated by comparisons between $W$ and $W^{\prime}$. (xlsx 
Additional file 3: Table S3. Statistics of GO enrichment analysis. (xlsx 55kb)

Additional file 4: Table S4. Statistics of Pathway enrichment analysis. (xlsx 14kb)

\section{Figures}
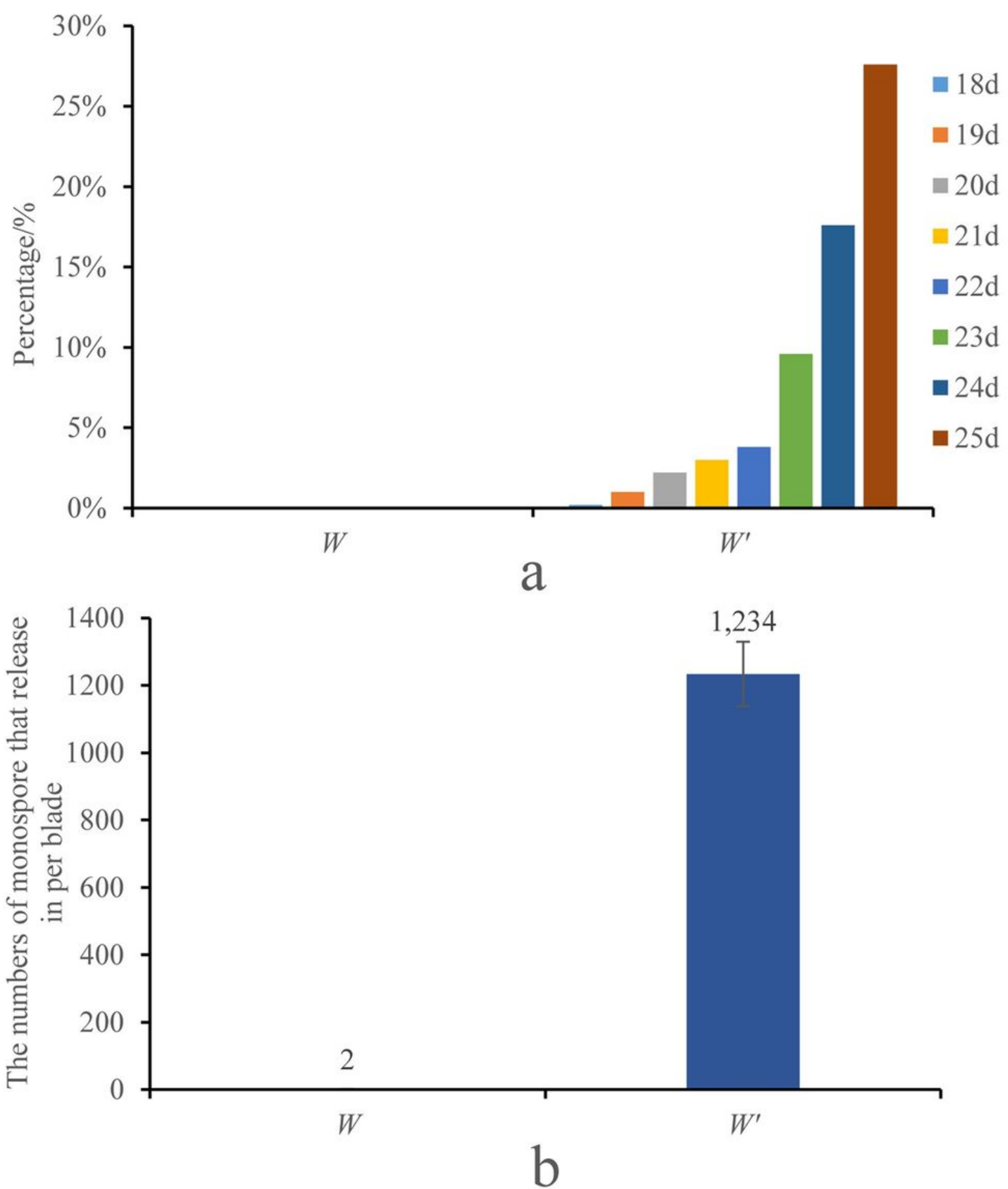

Figure 1 
Comparison of age point and ability of monospores' release between gametophytes of W and W'. a Percentage of gametophytes that release monospores between $\mathrm{W}$ and $\mathrm{W}^{\prime}$. b Total numbers of monospores that release in per blade between $\mathrm{W}$ and $\mathrm{W}^{\prime}$.

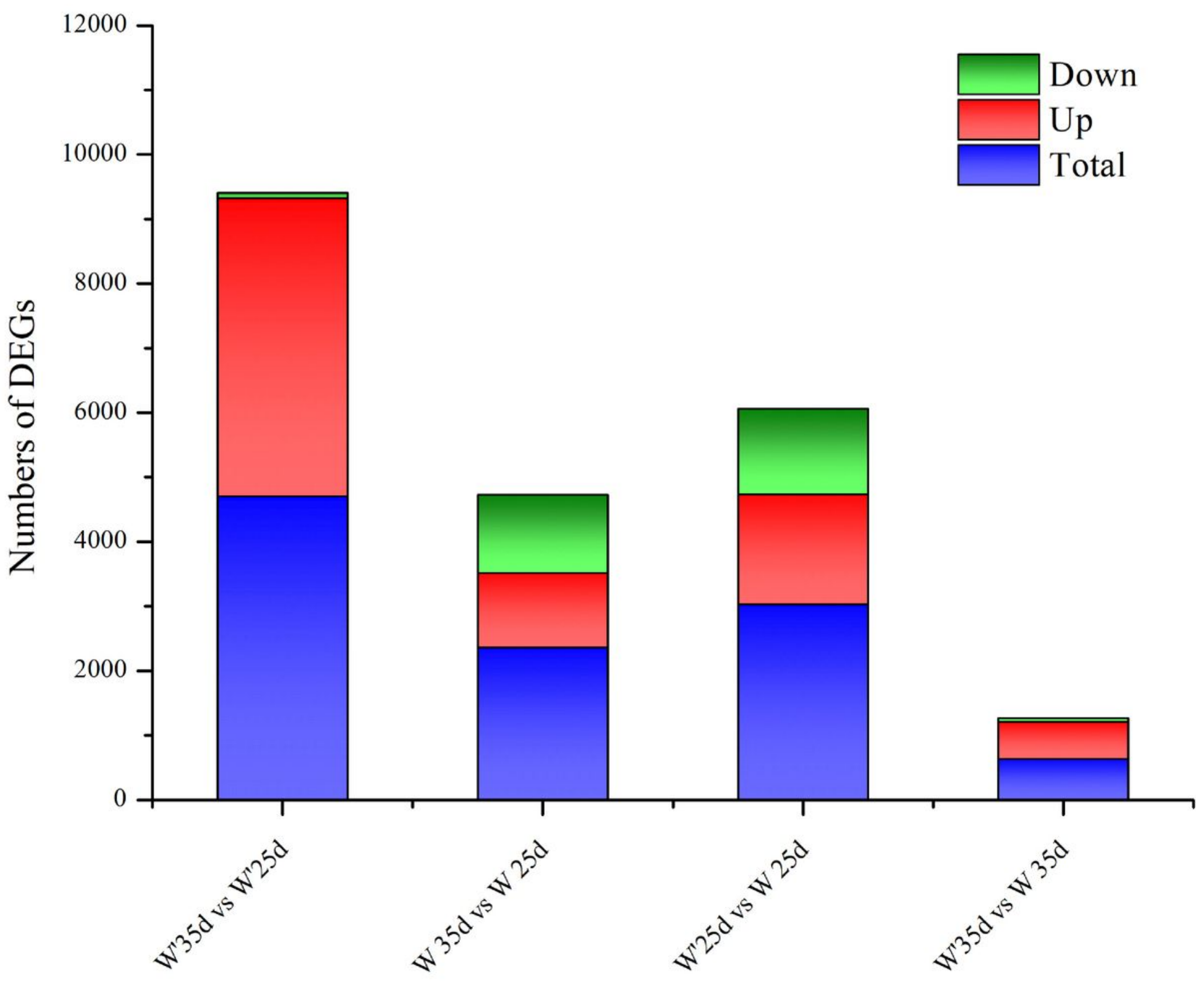

Figure 2

Statistics of the numbers of DEGs in different comparisons. 


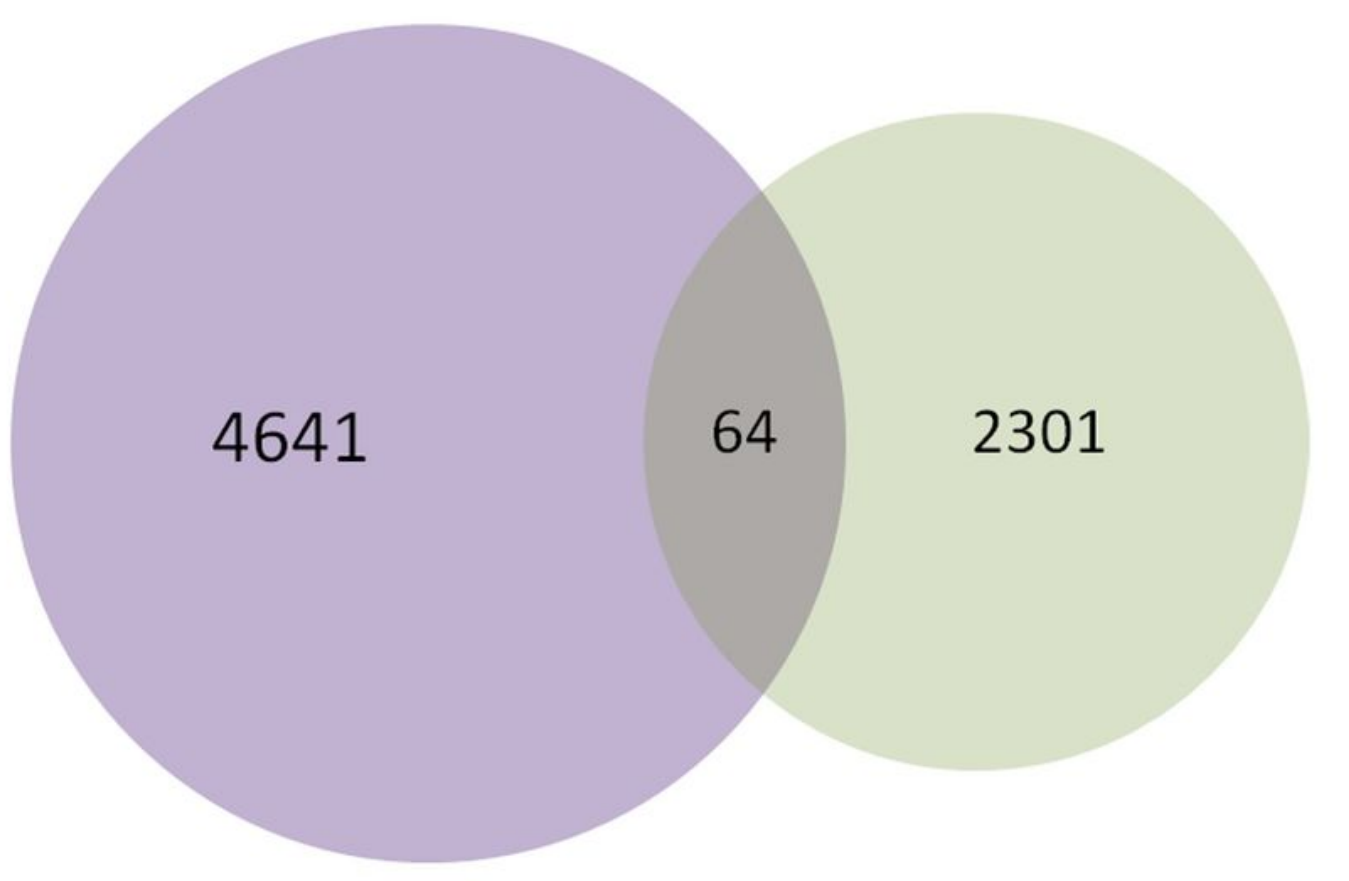

$W^{\prime} 35 \mathrm{~d}$ vs $W^{\prime} 25 \mathrm{~d} \quad W 35 \mathrm{~d}$ vs $W 25 \mathrm{~d}$

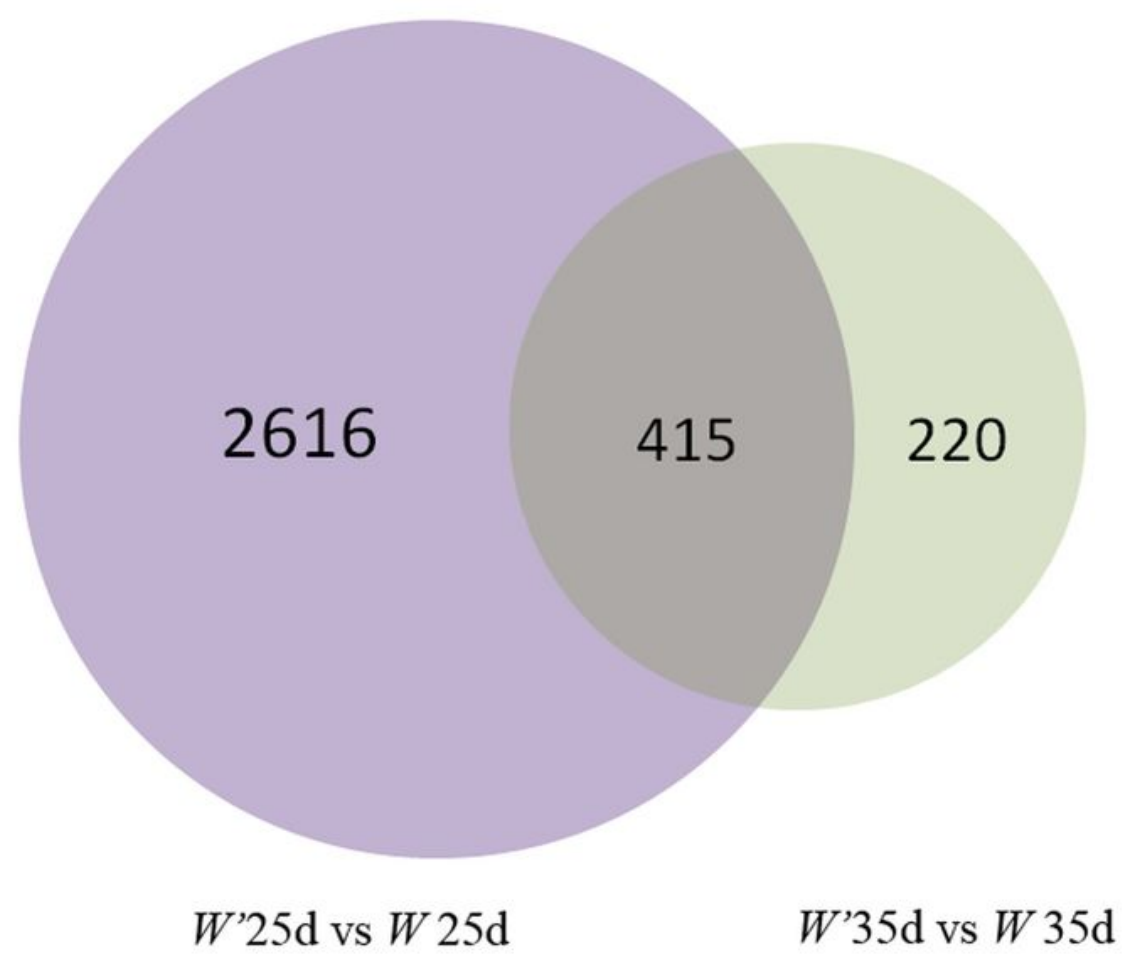

Figure 3

Venn analysis of DEGs. The sum of the numbers in the circle represents all of the DEGs produced in each comparison; the numbers in the overlapping parts represent the common DEGs between each comparison. 


\section{Top 20 of GO Enrichment}

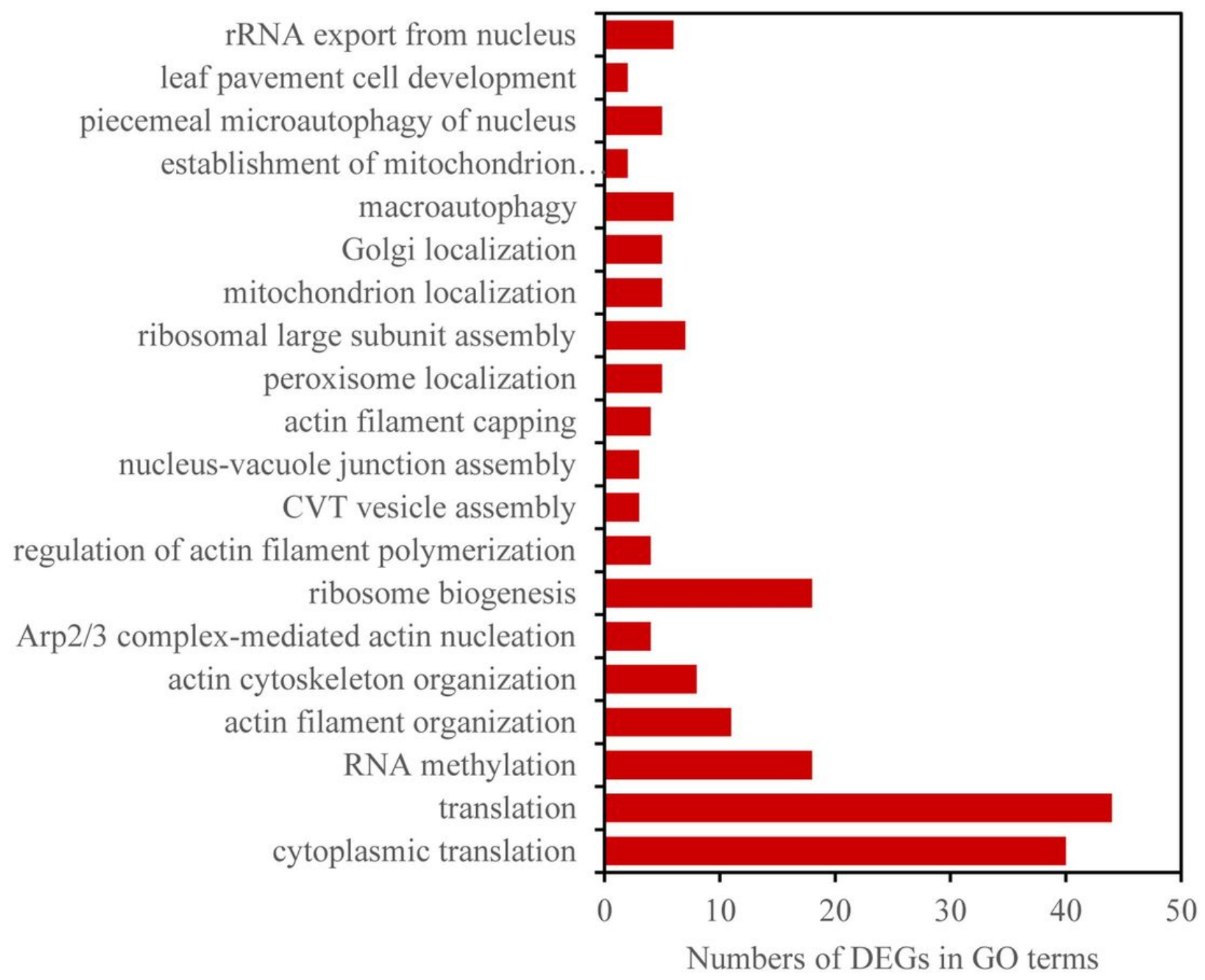

Figure 4

GO enrichment results of DEGs between W and W' on the same time point comparisons. 


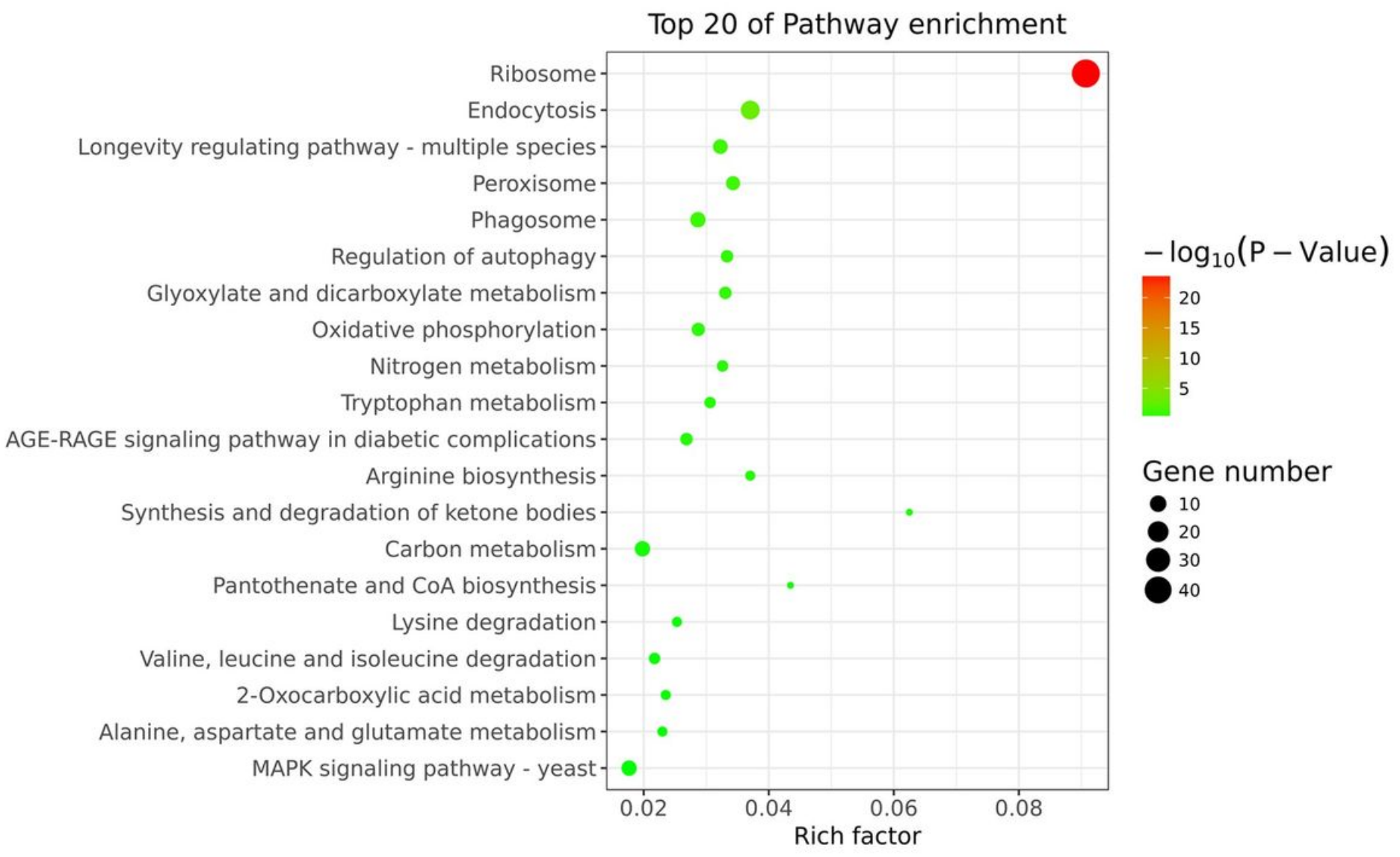

\section{Figure 5}

Pathway enrichment results of DEGs between W and W' on the same time point comparisons. 


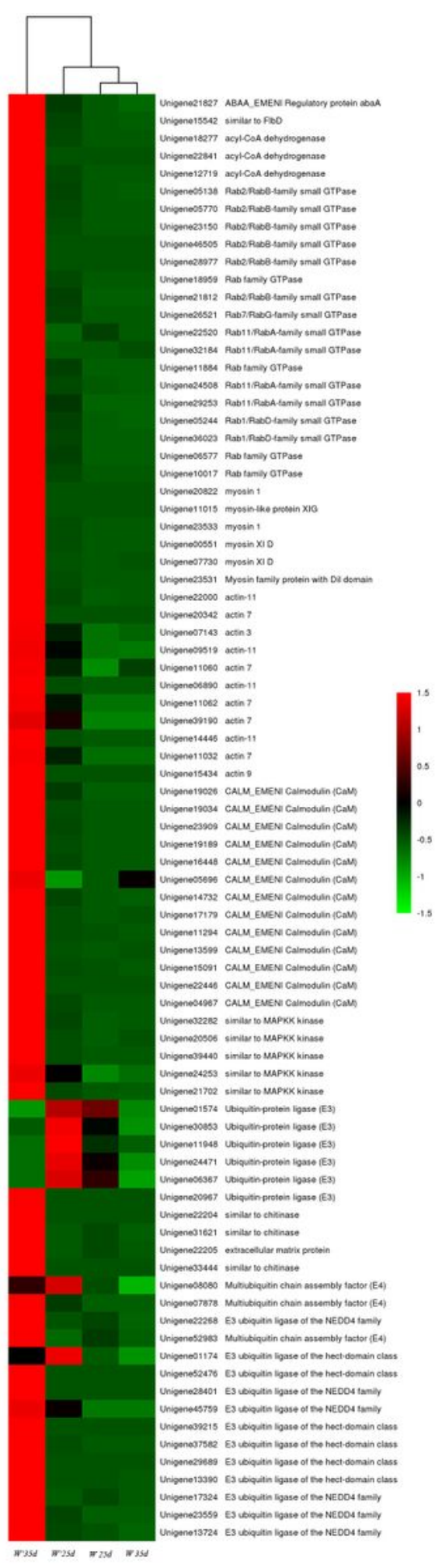

Figure 6

Hierarchical clustering of the expression values of partial DEGs in each sample. Red part represents high expression values and green part represents low expression values. Unigene represents Contig in this article. 

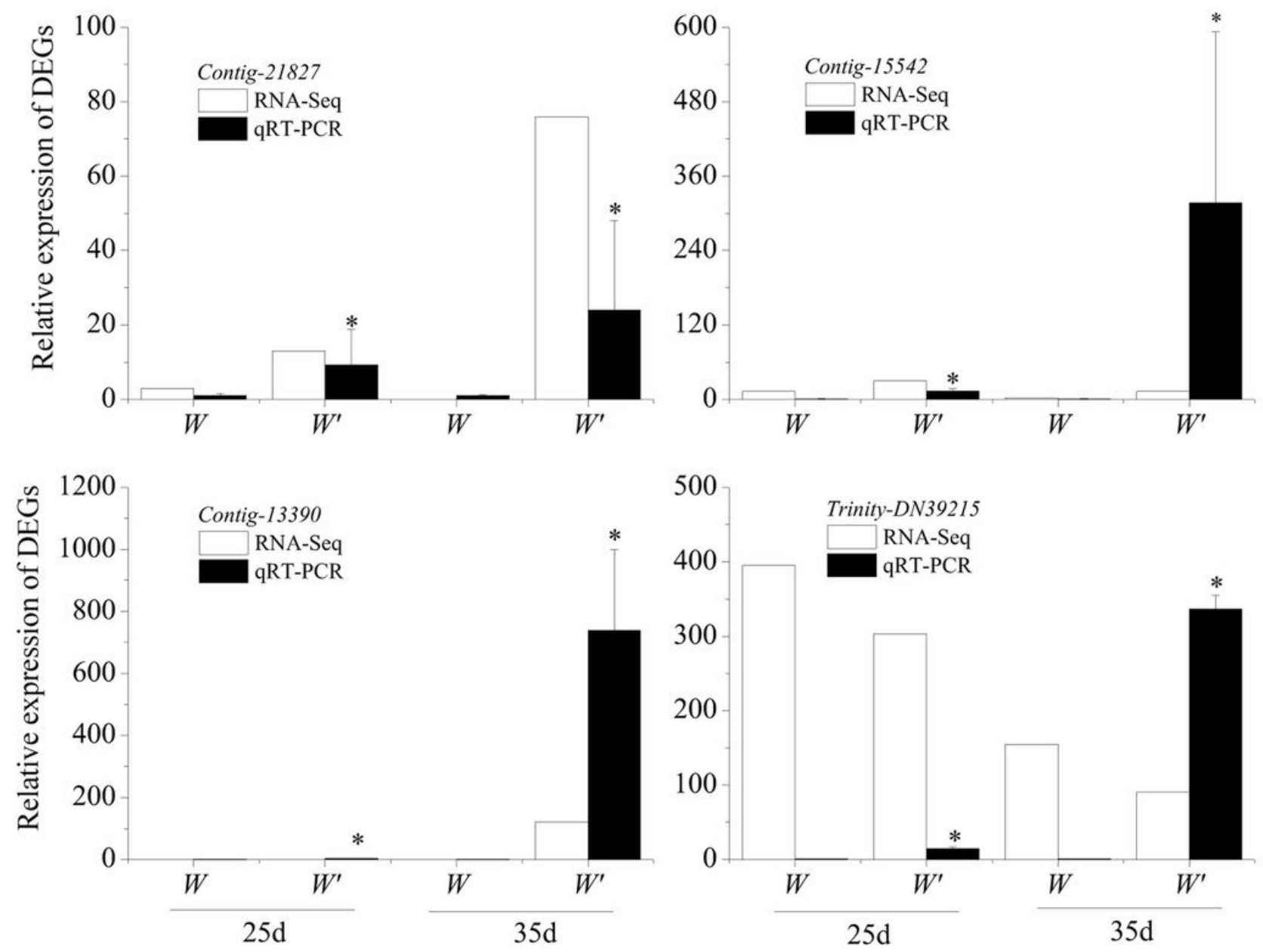

Figure 7

Relative expression analysis about selected DEGs by qRT-PCR between W and W'. " $\star$ " represents significant difference with control $(P<0.05)$. 


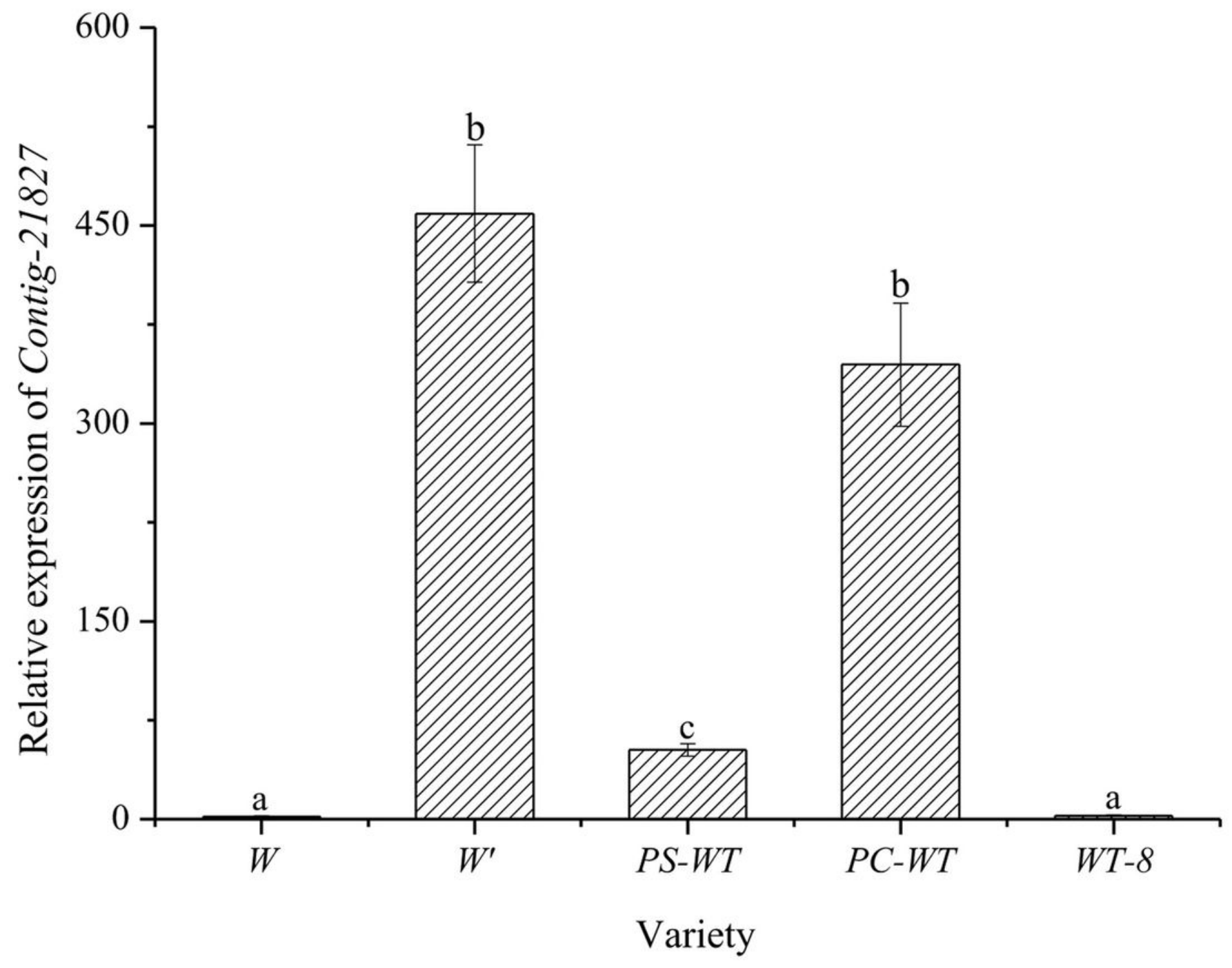

Figure 8

Relative expression of Contig-21827 in different strains and varieties of Pyropia on 35 day. a, b, c represents significant difference with control $(P<0.05)$.

\section{Supplementary Files}

This is a list of supplementary files associated with this preprint. Click to download.

- Additionalfile2.xlsx

- Additionalfile1.xlsx

- Additionalfile3.xlsx

- Additionalfile4.xlsx 